\title{
Derivation of relativistic Yakubovsky equations under Poincaré invariance
}

\author{
Hiroyuki Kamada ${ }^{\star}$ \\ Department of Physics, Faculty of Engineering, Kyushu Institute of Technology, \\ Kitakyushu 804-8550, Japan \\ $\star$ kamada@mns.kyutech.ac.jp \\ Proceedings for the 24th edition of European Few Body
Surrey, UK, 2-6 September 2019
doi:10.21468/SciPostPhysProc.3
}

\begin{abstract}
Relativistic Faddeev-Yakubovsky four-nucleon scattering equations are derived including a 3-body force. We present these equations in the momentum space representation. The quadratic integral equations using the iteration method, in order to obtain boosted potentials and 3-body force, are demonstrated.
\end{abstract}

(c) (1) Copyright H. Kamada

This work is licensed under the Creative Commons

Attribution 4.0 International License.

Published by the SciPost Foundation.
Received 29-10-2019

Accepted 08-01-2020

Published 24-02-2020

doi:10.21468/SciPostPhysProc.3.003

\section{Introduction}

At high energies one could expect deficiencies in the nonrelativistic Faddeev approach $[1,2]$ in three-nucleon system. We have been constructing a relativistic framework in the form of relativistic Faddeev equations [3-10] according to the Bakamjian-Thomas theory [11]. Not only using the realistic nonrelativistic nucleon-nucleon (NN) potentials but using Kharkov relativistic NN potential [12] we obtained the triton wave function by solving the relativistic Faddeev equation [13-15]. However, in the three-body scattering states, the relativistic effects appear to be generally small $[16,17]$ and insufficient to significantly improve the data description. For sensitive observations such as $A_{y}$ puzzles, the relativistic effect has certainly surfaced [18], but the results obtained were in the direction of deterioration.

As the number of particles handled increases, subtle relativistic effects will be accumulated and surface, so here we would like to rewrite the Yakubovsky equations [19], which solve the four-nucleon system exactly, to a relativistic equations as well.

In Section 1 we organize relativistic momenta and its Jacobian. Section 2 deals with rewriting interaction to relativistic potential by Lorentz boost. The boosted potential satisfies the relativistic Lippmann-Schwinger (LS) equation in Sec 3. Section 4 looks back on how the 3body Faddeev equation was relativistically transformed using the boosted potential. In Section 5 , we will remodel the four-body Yakubovsky equation and derive the relativistic Yakubovsky equation. In section 6, we derive relativistic equations involving 3-body force. A summary is in section 7. 


\section{2-body center of mass system}

In 2-body systems, these static mass of the particle are given $m_{i}(i=1,2)$. The four dimensional intrinsic momenta $\mathfrak{p}_{i}$ are

$$
\mathfrak{p}_{i}=\left(p_{i}^{\mu}\right)=\left(p_{i}^{0}, \vec{p}_{i}\right)=\left(E_{i}\left(p_{i}\right), p_{i}^{x}, p_{i}^{y}, p_{i}^{z}\right),
$$

with

$$
E_{i}\left(p_{i}\right)=\sqrt{m_{i}^{2}+p_{i}^{2}}=\sqrt{m_{i}^{2}+\vec{p}_{i} \cdot \vec{p}_{i}} .
$$

By Lorentz transformation $L=\left(L_{\mu}^{v}\right)$ of boosting velocity $\vec{v}$ the transformed four-momenta $\overline{\mathfrak{p}_{i}}$ are obtained

$$
\overline{\mathfrak{p}_{i}}=L \mathfrak{p}_{i}=\left(\overline{E_{i}}, \overline{\vec{p}_{i}}\right)=\left(\gamma\left(E_{i}-\vec{p}_{i} \cdot \vec{v}\right), \vec{p}_{i}+(\gamma-1)\left(\vec{p}_{i} \cdot \hat{v}\right) \hat{v}-\gamma E_{i} \vec{v}\right)
$$

with

$$
\gamma \equiv \frac{1}{\sqrt{1-v^{2}}}
$$

where $\hat{v}$ is the unit vector of $\vec{v}$ and light velocity is set to 1 .

The relativistic total momentum $\mathfrak{P}_{12}$ and the relative momentum $\vec{k}_{12}$ are given [20] as

$$
\begin{gathered}
\mathfrak{P}_{12} \equiv \mathfrak{p}_{1}+\mathfrak{p}_{2}=\left(E_{1}+E_{2}, \vec{p}_{1}+\vec{p}_{2}\right)=\left(E_{12}, \vec{P}_{12}\right), \\
\vec{k}_{12} \equiv \frac{\epsilon_{2} \vec{p}_{1}-\epsilon_{1} \vec{p}_{2}}{\epsilon_{1}+\epsilon_{2}},
\end{gathered}
$$

with

$$
\epsilon_{i} \equiv \frac{1}{2}\left(E_{i}+o_{i}\right)
$$

and

$$
o_{i} \equiv \sqrt{m_{i}^{2}+k_{12}^{2}}
$$

where we need pay attention that these equations from Eq.(6) to Eq. (8) are coupled for $k_{12}$. The momentum $\vec{k}_{12}$ is regarded an instanteneous momentum of center of mass system.

We start to enter the center of mass system, which the boosting velocity $\vec{u}$ is now chosen instead of $\vec{v}$

$$
\vec{u} \equiv \frac{\vec{p}_{1}+\vec{p}_{2}}{E_{1}+E_{2}}
$$

We have

$$
L \mathfrak{p}_{1}=\left(o_{1}, \vec{k}_{12}\right)
$$

and

$$
L \mathfrak{p}_{2}=\left(o_{2},-\vec{k}_{12}\right) .
$$

Therefore, we solve them to have $\vec{k}$ as

$$
\vec{k}_{12}=\frac{1}{2}\left(\left(\vec{p}_{1}-\vec{p}_{2}\right)-\left(\vec{p}_{1}+\vec{p}_{2}\right)\left(\frac{E_{1}-E_{2}+\frac{m_{1}^{2}-m_{2}^{2}}{\sqrt{\left(E_{1}+E_{2}\right)^{2}-\left(\vec{p}_{1}+\vec{p}_{2}\right)^{2}}}}{E_{1}+E_{2}+\sqrt{\left(E_{1}+E_{2}\right)^{2}-\left(\vec{p}_{1}+\vec{p}_{2}\right)^{2}}}\right)\right) .
$$


We know the following Jacobian $\mathcal{J}_{12}$

$$
\mathcal{J}_{12} \equiv \frac{\partial\left(\vec{p}_{1}, \vec{p}_{2}\right)}{\partial(\vec{k}, \vec{P})}=\frac{E_{1} E_{2}}{E_{1}+E_{2}} \frac{o_{1}+o_{2}}{o_{1} o_{2}} .
$$

If we take the case of equal mass $\left(m_{1}=m_{2}\right)$, we have $o_{1}=o_{2}$,

$$
\left.\vec{k}_{12}\right|_{m_{1}=m_{2}}=\frac{1}{2}\left(\left(\vec{p}_{1}-\vec{p}_{2}\right)-\left(\vec{p}_{1}+\vec{p}_{2}\right)\left(\frac{E_{1}-E_{2}}{E_{1}+E_{2}+\sqrt{\left(E_{1}+E_{2}\right)^{2}-\left(\vec{p}_{1}+\vec{p}_{2}\right)^{2}}}\right)\right)
$$

and

$$
\left.\mathcal{J}_{12}\right|_{m_{1}=m_{2}}=\frac{E_{1} E_{2}}{E_{1}+E_{2}} \frac{4}{\sqrt{\left(E_{1}+E_{2}\right)^{2}-\left(\vec{p}_{1}+\vec{p}_{2}\right)^{2}}} .
$$

Eq. (14) and Eq. (15) are corresponding to Eq.(3.6) and (3.16) of [3], respectively.

\section{Boosted potential}

Let us consider two equal mass particles $\left(m=m_{1}=m_{2}\right)$ which are labeled 1 and 2 in the 2-body center of mass system with interaction $v_{12}$. We have a invariant mass $\sqrt{S_{12}}$ of the system as

$$
\sqrt{S_{12}}=2 \sqrt{m^{2}+k_{12}^{2}}+v_{12},
$$

where $k_{12}$ is the relative momentum between particle 1 and 2 .

On the other hand, we leave from the 2-body c. m. system, the total momentum $\vec{P}_{12} \equiv \vec{p}_{1}+\vec{p}_{2}$ is nonzero. The invariant mass $\sqrt{S_{12}^{\text {boost }}}$ is given as

$$
\sqrt{S_{12}^{\text {boost }}}=\sqrt{S_{12}+P_{12}^{2}}=\sqrt{\left(2 \sqrt{m^{2}+k_{12}^{2}}+v_{12}\right)^{2}+P_{12}^{2}} .
$$

Now, one introduce so-called boosted potential $V_{12}$ as

$$
\begin{aligned}
V_{12}\left(P_{12}\right) & \equiv \sqrt{s_{12}^{\text {boost }}}-\sqrt{\left(2 \sqrt{m^{2}+k_{12}^{2}}+0\right)^{2}+P_{12}^{2}} \\
& =\sqrt{\left(2 \sqrt{m^{2}+k_{12}^{2}}+v_{12}\right)^{2}+P_{12}^{2}}-\sqrt{4\left(m^{2}+k_{12}^{2}\right)+P_{12}^{2}}
\end{aligned}
$$

After quantumization $\left(k_{12} \rightarrow \hat{k}_{12}, v \rightarrow \hat{v}\right.$ and $\left.V \rightarrow \hat{V}\right)$ the boosted potential operator $\hat{V}_{12}\left(P_{12}\right)$ is still a diagonal operator to the boosting momentum $P_{12}$. We have a boosted Schrödinger equation for the wave function $\phi_{12}$

$$
\left(\sqrt{4\left(m^{2}+\hat{k}_{12}^{2}\right)+P_{12}^{2}}+\hat{V}_{12}\left(P_{12}\right)\right) \phi_{12}=\sqrt{M^{2}+P_{12}^{2}} \phi_{12}
$$

and a un-boosted one,

$$
\left(2 \sqrt{m^{2}+\hat{k}_{12}^{2}}+\hat{v}_{12}\right) \phi_{12}=M \phi_{12},
$$

where $M$ is a eigen value of mass operator $\sqrt{\hat{S}_{12}}$. 


\section{Relativistic Faddeev Equations}

For the 3-body system, we add to third equal mass particle. There are 3 piarwises (subsystems) denoted not only as (12) but as (23) and (31). For each piarwise (ij) we can define the boosted potential as Eq. (18) by the boosting momentum $\vec{P}_{i j}$.

$$
V_{i j}\left(P_{i j}\right) \equiv \sqrt{\left(2 \sqrt{m^{2}+k_{i j}^{2}}+v_{i j}\right)^{2}+P_{i j}^{2}}-\sqrt{4\left(m^{2}+k_{i j}^{2}\right)+P_{i j}^{2}} .
$$

We choose now the boosting momentum $\vec{P}_{i j}=-\vec{p}_{k}$, which means it is 3-body c. m. system $(i \neq k \neq j)$;

$$
\vec{P}_{i j}=\vec{p}_{i}+\vec{p}_{j}=-\vec{p}_{k}, \quad \vec{p}_{1}+\vec{p}_{2}+\vec{p}_{3}=0 .
$$

One may naturally have an idea the following 3-body invariant mass $\sqrt{S_{123}}$ poses a symmetry.

$$
\begin{aligned}
\sqrt{S_{123}} & =\sqrt{m^{2}+p_{1}^{2}}+\sqrt{m^{2}+p_{2}^{2}}+\sqrt{m^{2}+p_{3}^{2}}+V_{12}\left(P_{12}\right)+V_{23}\left(P_{23}\right)+V_{31}\left(P_{31}\right) \\
& =\sqrt{m^{2}+p_{1}^{2}}+\sqrt{m^{2}+p_{2}^{2}}+\sqrt{m^{2}+p_{3}^{2}}+V_{12}\left(p_{3}\right)+V_{23}\left(p_{1}\right)+V_{31}\left(p_{2}\right) \\
& =\sqrt{\left(2 \sqrt{m^{2}+k_{12}^{2}}+v_{12}\right)^{2}+p_{3}^{2}}+\sqrt{m^{2}+p_{3}^{2}}+V_{23}\left(p_{1}\right)+V_{31}\left(p_{2}\right) \\
& =\sqrt{\left(2 \sqrt{m^{2}+k_{23}^{2}}+v_{23}\right)^{2}+p_{1}^{2}}+\sqrt{m^{2}+p_{1}^{2}}+V_{31}\left(p_{2}\right)+V_{12}\left(p_{3}\right) \\
& =\sqrt{\left(2 \sqrt{m^{2}+k_{31}^{2}}+v_{31}\right)^{2}+p_{2}^{2}}+\sqrt{m^{2}+p_{2}^{2}}+V_{12}\left(p_{3}\right)+V_{23}\left(p_{1}\right) .
\end{aligned}
$$

This symmetry helps us to build the relativistic Faddeev equations. After the quantumization we write the relativistic Faddeev equation for bound state as

$$
\phi_{i j}=\hat{G}_{0} \hat{t}_{i j}\left(\phi_{j k}+\phi_{k i}\right) \text {, }
$$

where $\phi_{i j}$ is the Faddeev component for the subsystem (ij)

$$
\phi_{i j} \equiv \hat{G}_{0} \hat{V}_{i j} \Psi
$$

with the total wave function $\Psi$

$$
\Psi=\phi_{12}+\phi_{23}+\phi_{31}
$$

and $\hat{G}_{0}$ is the three-body Green's function,

$$
\hat{G}_{0}=\frac{1}{M_{123}-\left(\sqrt{4\left(m^{2}+\hat{k}_{i j}^{2}\right)+\hat{p}_{k}^{2}}+\sqrt{m^{2}+\hat{p}_{k}^{2}}\right)}
$$

and $\hat{t}_{i j}$ is the t-matrix of subsystem (ij) which is satisfactory with the LS equation;

$$
\hat{t}_{i j}=\hat{V}_{i j}+\hat{V}_{i j} \hat{G}_{0} \hat{t}_{i j}
$$

where $M_{123}$ is the eigen value of the mass operator $\sqrt{\hat{S}_{123}}$.

In the case of a system with identical particles, the permutation operators built from transpositions $\mathcal{P}_{i j}$, interchanging particles $i$ and $j$, are used to express all two-body interaction

$$
V_{12}+V_{23}+V_{31} \equiv\left(1+\mathcal{P}_{12} \mathcal{P}_{23}+\mathcal{P}_{13} \mathcal{P}_{23}\right) V_{12}=(1+\mathcal{P}) V,
$$

where we have singled out the (12) pair and denoted $V \equiv V_{12}, t \equiv t_{12}$ and $\phi_{12} \equiv \phi$ with permutation operator $\mathcal{P} \equiv \mathcal{P}_{12} \mathcal{P}_{23}+\mathcal{P}_{13} \mathcal{P}_{23}$. We have a simple presentation of Eq. (24).

$$
\phi=\hat{G}_{0} \hat{t} \mathcal{P} \phi .
$$




\section{Relativistic Yakubovsky Equations}

For the 4-body system, we add to fourth equal mass particle. There are 6 piarwises (subsystems) denoted not only as (12), (23) and (31) but as (14),(24) and (34).

We choose now the boosting momentum $\vec{P}_{i j}=-\vec{p}_{k}-\vec{p}_{l}$, which means it is 4-body c. $\mathrm{m}$. system $(k \neq i, j, l$, and $l \neq i, j, k)$;

$$
\vec{P}_{i j}=\vec{p}_{i}+\vec{p}_{j}=-\vec{p}_{k}-\vec{p}_{l}=-\vec{P}_{k l}, \quad \vec{p}_{1}+\vec{p}_{2}+\vec{p}_{3}+\vec{p}_{4}=0 .
$$

One may naturally have an idea the following 4-body invariant mass $\sqrt{S_{1234}}$ poses a symmetry.

$$
\begin{aligned}
\sqrt{S_{1234}} & =\sqrt{m^{2}+p_{1}^{2}}+\sqrt{m^{2}+p_{2}^{2}}+\sqrt{m^{2}+p_{3}^{2}}+\sqrt{m^{2}+p_{4}^{2}} \\
& +V_{12}\left(P_{34}\right)+V_{23}\left(P_{14}\right)+V_{31}\left(P_{24}\right)+V_{14}\left(P_{23}\right)+V_{24}\left(P_{31}\right)+V_{34}\left(P_{12}\right) .
\end{aligned}
$$

The most important thing is that during generate the boosting potential $V_{i j}$ the momentum $P_{k l}$ behaves as a parameter. In other word, the boosting potential operator $\hat{V}_{i j}$ is diagonal to the momentum $P_{k l}$.

Using the 3-body relative momentum $\vec{q}_{k}$ between the subsystem (ij) and the third particle, and the [2+2] partition relative momentum $\vec{s}_{l}$ between the subsystem (ij) and (kl) (see Apppendix A) we rewrite the 4-body invaliant mass $\sqrt{S_{1234}}$

$$
\begin{aligned}
& \sqrt{S_{1234}}=\quad=\sqrt{\left(\sqrt{\left(2 \sqrt{m^{2}+k_{12}^{2}}\right)^{2}+q_{3}^{2}}+\sqrt{m^{2}+q_{3}^{2}}\right)^{2}+p_{4}^{2}}+\sqrt{m^{2}+p_{4}^{2}} \\
& +V_{12}^{[3+1]}\left(q_{3} ; p_{4}\right)+V_{23}+V_{31}+V_{14}+V_{24}+V_{34} \\
& =\sqrt{\left(\sqrt{\left(2 \sqrt{m^{2}+k_{12}^{2}}+v_{12}\right)^{2}+q_{3}^{2}}+\sqrt{m^{2}+q_{3}^{2}}\right)^{2}+p_{4}^{2}}+\sqrt{m^{2}+p_{4}^{2}} \\
& +V_{23}+V_{31}+V_{14}+V_{24}+V_{34} \\
& =\sqrt{\left(2 \sqrt{m^{2}+k_{12}^{2}}\right)^{2}+s_{4}^{2}}+\sqrt{\left(2 \sqrt{m^{2}+k_{34}^{2}}\right)^{2}+s_{4}^{2}} \\
& +V_{12}^{[2+2]}\left(s_{4}\right)+V_{23}+V_{31}+V_{14}+V_{24}+V_{34} \\
& =\sqrt{\left(2 \sqrt{m^{2}+k_{12}^{2}}+v_{12}\right)^{2}+s_{4}^{2}}+\sqrt{\left(2 \sqrt{m^{2}+k_{34}^{2}}\right)^{2}+s_{4}^{2}} \\
& +V_{23}+V_{31}+V_{14}+V_{24}+V_{34} \text {, }
\end{aligned}
$$

where $q_{3}$ is a relative momentum between subsystem (12) and the third particle, and $s_{4}$ is a relative momentum between subsystem (12) and (34). (see Appendix A)

The following equations from (34) to (43) are simply demonstrated as usual nonrelativistic Yakubovsky form except for the relativistic Green's function (39) and the boosted potential. Similarily (25), Faddeev component $\phi_{i j}^{(4)}$ is defined with the four-body total wave function $\Psi^{(4)}$ as

$$
\phi_{i j}^{(4)} \equiv \hat{G}_{0}^{(4)} \hat{V}_{i j} \Psi^{(4)}
$$

where $\Psi^{(4)}$ is the total wave function which consists of 6 Faddeev components $\phi_{i j}^{(4)}$

$$
\Psi^{(4)}=\sum_{(i j)} \phi_{i j}^{(4)}
$$

In case of identical particle, using permutation operator $\mathcal{P}, \mathcal{P}_{34}$ and $\tilde{\mathcal{P}}$ we have Faddeev equations for the four-body system as

$$
\phi^{(4)}=\phi_{12}^{(4)}=\hat{G}_{0}^{(4)} \hat{t}^{(4)}\left(\mathcal{P}-\mathcal{P}_{34} \mathcal{P}+\tilde{\mathcal{P}}\right) \phi^{(4)} \equiv\left(1-\mathcal{P}_{34}\right) \psi_{1}+\psi_{2},
$$


with

$$
\tilde{\mathcal{P}} \equiv \mathcal{P}_{13} \mathcal{P}_{24},
$$

where we have again singled out the (12) pair and denoted $V \equiv V_{12}, t^{(4)} \equiv t_{12}^{(4)}$ and $\phi_{12}^{(4)} \equiv \phi^{(4)}$, and $\hat{t}^{(4)}$ also obeys Lippmann-Schwinger equation;

$$
\hat{t}^{(4)}=\hat{V}+\hat{V} \hat{G}_{0}^{(4)} \hat{t}^{(4)}
$$

and $\hat{G}_{0}^{(4)}$ is the four-body Green's function,

$$
\begin{aligned}
\hat{G}_{0}^{(4)}= & \frac{1}{M_{1234}-\left(\sqrt{\left(\sqrt{\left(2 \sqrt{m^{2}+\hat{k}_{12}^{2}}\right)^{2}+\hat{q}_{3}^{2}}+\sqrt{m^{2}+\hat{q}_{3}^{2}}\right)^{2}+\hat{p}_{4}^{2}}+\sqrt{m^{2}+\hat{p}_{4}^{2}}\right)} \\
= & \frac{1}{M_{1234}-\left(\sqrt{\left(2 \sqrt{m^{2}+\hat{k}_{12}^{2}}\right)^{2}+\hat{s}_{4}^{2}}+\sqrt{\left(2 \sqrt{m^{2}+\hat{k}_{34}^{2}}\right)^{2}+\hat{s}_{4}^{2}}\right)} .
\end{aligned}
$$

The Yakubovsky components $\psi_{1}$ and $\psi_{2}$ already appear in Eq.(36) which are defined as

$$
\begin{aligned}
& \psi_{1} \equiv \hat{G}_{0}^{(4)} \hat{t}^{(4)} \mathcal{P} \phi^{(4)}, \\
& \psi_{2} \equiv \hat{G}_{0}^{(4)} \hat{t}^{(4)} \tilde{\mathcal{P}} \phi^{(4)} .
\end{aligned}
$$

We show the relativistic Yakubovsky equations for bound state.

$$
\begin{aligned}
& \psi_{1}=-\hat{G}_{0}^{(4)} \hat{T} \mathcal{P}_{34} \psi_{1}+\hat{G}_{0}^{(4)} \hat{T} \psi_{2}, \\
& \psi_{2}=\hat{G}_{0}^{(4)} \hat{\tilde{T}} \tilde{\mathcal{P}}\left(1-\mathcal{P}_{34}\right) \psi_{1},
\end{aligned}
$$

where $\hat{T}$ and $\hat{\tilde{T}}$ are 3-body t-matrix operator and $2+2$ partition t-matrix operator, respectively.

$$
\begin{aligned}
& \hat{T}=\hat{t}^{(4)}+\hat{t}^{(4)} \mathcal{P} \hat{G}_{0}^{(4)} \hat{T}, \\
& \hat{\tilde{T}}=\hat{t}^{(4)}+\hat{t}^{(4)} \tilde{\mathcal{P}} \hat{G}_{0}^{(4)} \hat{\tilde{T}} .
\end{aligned}
$$

The relativistic Yakubovsky equation (42) keeps similar form as nonrelativistic one.

\section{Inclusion of 3-body force}

Recently [21,22], we obtained the Faddeev equations and Yakubovsky equations including 3-body force. The 3-body force $w_{123}$ is naturally decomposed into three parts

$$
w_{123} \equiv w_{123}^{(1)}+w_{123}^{(2)}+w_{123}^{(3)}=(1+\mathcal{P}) w_{123}^{(3)}=(1+\mathcal{P}) w
$$

with $w=w_{123}^{(3)}$. Instead of Eq. (25) the Faddeev component is defined including a part of 3-body force

$$
\phi=\hat{G}_{0}(\hat{V}+\hat{w}) \Psi
$$

Including 3-body force the Faddeev equation for bound state is rewritten as

$$
\phi=\hat{G}_{0} \hat{\tau} \phi
$$


where $\hat{\tau}$ is defined as

$$
\hat{\tau} \equiv \hat{t} \mathcal{P}+\left(1+\hat{t} \hat{G}_{0}\right) \hat{w}(1+\mathcal{P})
$$

Because the 3-body force are given in 3-body center of mass system, we need not boost the 3-body force.

On the other hand, in case of the 4-body system, the 3-body force $\hat{w}_{123}$ is required to be boosted with the direction of the last momentum $\vec{p}_{4}$. The invariant mass of the 4-body system $\sqrt{S_{1234}}$ may be given as

$$
\begin{aligned}
\sqrt{S_{1234}} & =\sqrt{m^{2}+p_{1}^{2}}+\sqrt{m^{2}+p_{2}^{2}}+\sqrt{m^{2}+p_{3}^{2}}+\sqrt{m^{2}+p_{4}^{2}} \\
& +V_{12}+V_{23}+V_{31}+V_{14}+V_{24}+V_{34} \\
& +W_{123}\left(p_{4}\right)+W_{234}\left(p_{1}\right)+W_{341}\left(p_{2}\right)+W_{412}\left(p_{3}\right) \\
& =\sqrt{m^{2}+p_{1}^{2}}+\sqrt{m^{2}+p_{2}^{2}}+\sqrt{m^{2}+p_{3}^{2}}+\sqrt{m^{2}+p_{4}^{2}} \\
& +V_{12}+V_{23}+V_{31}+V_{14}+V_{24}+V_{34} \\
& +W_{123}^{(1)}\left(p_{4}\right)+W_{234}^{(2)}\left(p_{1}\right)+W_{341}^{(3)}\left(p_{2}\right)+W_{412}^{(4)}\left(p_{3}\right) \\
& +W_{123}^{(2)}\left(p_{4}\right)+W_{234}^{(3)}\left(p_{1}\right)+W_{341}^{(4)}\left(p_{2}\right)+W_{412}^{(1)}\left(p_{3}\right) \\
& +W_{123}^{(3)}\left(p_{4}\right)+W_{234}^{(4)}\left(p_{1}\right)+W_{341}^{(1)}\left(p_{2}\right)+W_{412}^{(2)}\left(p_{3}\right) \\
& =\sqrt{\left(\sqrt{4\left(m^{2}+k_{12}^{2}\right)+q_{3}^{2}}+\sqrt{m^{2}+q_{3}^{2}}+w_{123}^{(3)}\right)^{2}+p_{4}^{2}}+\sqrt{m^{2}+p_{4}^{2}} \\
& +V_{12}+V_{23}+V_{31}+V_{14}+V_{24}+V_{34} \\
& +W_{123}^{(1)}\left(p_{4}\right)+W_{234}^{(2)}\left(p_{1}\right)+W_{341}^{(3)}\left(p_{2}\right)+W_{412}^{(4)}\left(p_{3}\right) \\
& +W_{123}^{(2)}\left(p_{4}\right)+W_{234}^{(3)}\left(p_{1}\right)+W_{341}^{(4)}\left(p_{2}\right)+W_{412}^{(1)}\left(p_{3}\right) \\
& \quad+W_{234}^{(4)}\left(p_{1}\right)+W_{341}^{(1)}\left(p_{2}\right)+W_{412}^{(2)}\left(p_{3}\right),
\end{aligned}
$$

where $W_{i j k}^{(i)}\left(p_{l}\right), W_{i j k}^{(j)}\left(p_{l}\right)$ and $W_{i j k}^{(k)}\left(p_{l}\right)$ are boosted 3-body forces. (see Appendix B) Similarily, instead of Eq. (40) the Yakubovsky component is defined including a part of boosted 3-body force $\hat{W} \equiv \hat{W}_{123}^{(3)}$,

$$
\begin{aligned}
& \psi_{1} \equiv \hat{G}_{0}^{(4)} \hat{t}^{(4)} \mathcal{P} \phi^{(4)}+\left(1+\hat{G}_{0}^{(4)} \hat{t}^{(4)}\right) \hat{G}_{0}^{(4)} \hat{W} \Psi^{(4)}, \\
& \psi_{2} \equiv \hat{G}_{0}^{(4)} \hat{t}^{(4)} \tilde{\mathcal{P}} \phi^{(4)},
\end{aligned}
$$

where $\Psi^{(4)}$ is the total wave function

$$
\Psi^{(4)}=\left(1+\mathcal{P}-\mathcal{P}_{34} \mathcal{P}+\tilde{\mathcal{P}}\right)\left(\psi_{1}-\mathcal{P}_{34} \psi_{1}+\psi_{2}\right) .
$$

Yakubovsky 4-body equations for bound state are rewritten as

$$
\begin{aligned}
\psi_{1}=\quad & -\hat{G}_{0}^{(4)} \hat{\mathcal{T}} \mathcal{P}_{34} \psi_{1}+\hat{G}_{0}^{(4)} \hat{\mathcal{T}} \psi_{2} \\
& +\left(1+\hat{G}_{0}^{(4)} \hat{\mathcal{T}}\right)\left(1+\hat{G}_{0}^{(4)} \hat{t}^{(4)}\right) \hat{G}_{0}^{(4)} \hat{W}\left(-\mathcal{P}_{34} \mathcal{P}+\tilde{\mathcal{P}}\right)\left(\psi_{1}-\mathcal{P}_{34} \psi_{1}+\psi_{2}\right), \\
\psi_{2}= & \hat{G}_{0}^{(4)} \hat{\tilde{T}} \tilde{\mathcal{P}}\left(1-\mathcal{P}_{34}\right) \psi_{1}
\end{aligned}
$$

with

$$
\hat{\mathcal{T}}=\hat{\tau}^{(4)}+\hat{\tau}^{(4)} \hat{G}_{0}^{(4)} \hat{\mathcal{T}} .
$$

\section{Conclusion}

The relativistic Faddeev 3-body equations and the relativistic Yakubovsky 4-body equations are given in Eq.(30) and Eq.(42), respectively. These equations are not deformed from the original 
ones with the boosted potentials in Eq. (18) and Eq.(33). Inclusion of 3-body force is treated consistently in Faddeev equations (46) and Yakubovsky ones (51) under Poincaré invariance. These boosted potentials and the boosted 3-body force are defined by Eqs. (55), (58) and (59) in Appendices A and B. The quadratic integral equations using the iteration method [7], in order to obtain these boosted potentials and 3-body force, are demonstrated in Appendix C.

\section{Acknowledgements}

Author (H.K.) would like to thank H. Witała, J. Golak, R. Skibiński, K. Topolnicki, A. Nogga and E. Epelbaum for fruitful discussions during the 4th LENPIC meeting in Bochum, Germany. website http://www.lenpic.org/

\section{A Appendix A}

The relative momentum $\vec{q}_{3}$ between subsystem (12) and the third particle is given as

$$
\vec{q}_{3} \equiv \frac{\epsilon_{12} \vec{p}_{3}-\epsilon_{3} \vec{P}_{12}}{\epsilon_{12}+\epsilon_{3}}
$$

with

$$
\epsilon_{12}=\frac{1}{2}\left(E_{1}+E_{2}+\sqrt{\left(2 \sqrt{m^{2}+k_{12}^{2}}\right)^{2}+q_{3}^{2}}\right) .
$$

Using the momentum $\vec{q}_{3}$ we have the boosted potential $V_{12}^{[3+1]}\left(q_{3} ; p_{4}\right)$ in Eq. (33).

$$
\begin{aligned}
V_{12}^{[3+1]}\left(q_{3} ; p_{4}\right) \equiv & \sqrt{\left(\sqrt{\left(2 \sqrt{m^{2}+k_{12}^{2}}+v_{12}\right)^{2}+q_{3}^{2}}+\sqrt{m^{2}+q_{3}^{2}}\right)^{2}+p_{4}^{2}} \\
& -\sqrt{\left(\sqrt{\left(2 \sqrt{m^{2}+k_{12}^{2}}\right)^{2}+q_{3}^{2}}+\sqrt{m^{2}+q_{3}^{2}}\right)^{2}+p_{4}^{2}} .
\end{aligned}
$$

The [2+2] partition relative momentum $\vec{s}_{4}$ between subsystem (12) and (34) is given as

$$
\vec{s}_{4} \equiv \frac{\epsilon_{12,34} \vec{P}_{34}-\epsilon_{34,12} \vec{P}_{12}}{\epsilon_{12,34}+\epsilon_{34,12}},
$$

with

$$
\begin{aligned}
& \epsilon_{12,34}=\frac{1}{2}\left(E_{1}+E_{2}+\sqrt{\left(2 \sqrt{m^{2}+k_{12}^{2}}\right)^{2}+s_{4}^{2}}\right), \\
& \epsilon_{34,12}=\frac{1}{2}\left(E_{3}+E_{4}+\sqrt{\left(2 \sqrt{m^{2}+k_{34}^{2}}\right)^{2}+s_{4}^{2}}\right) .
\end{aligned}
$$

Using the momentum $\vec{s}_{4}$ we have the boosted potential $V_{12}^{[2+2]}\left(s_{4}\right)$ in Eq. (33).

$$
V_{12}^{[2+2]}\left(s_{4}\right) \equiv \sqrt{\left(2 \sqrt{m^{2}+k_{12}^{2}}+v_{12}\right)^{2}+s_{4}^{2}}-\sqrt{\left(2 \sqrt{m^{2}+k_{12}^{2}}\right)^{2}+s_{4}^{2}}=V_{12}\left(s_{4}\right) .
$$

Actually, this is the same definition of $V_{12}$ in Eq. (18). 


\section{B Appendix B}

The boosted 3-body force $W_{123}^{(3)}\left(p_{4}\right)$ is defined as

$$
\begin{aligned}
W_{123}^{(3)}\left(p_{4}\right) \equiv & \sqrt{\left(\sqrt{\left(2 \sqrt{m^{2}+k_{12}^{2}}\right)^{2}+q_{3}^{2}}+\sqrt{m^{2}+q_{3}^{2}}+w_{123}^{(3)}\right)^{2}+p_{4}^{2}} \\
& -\sqrt{\left(\sqrt{\left(2 \sqrt{m^{2}+k_{12}^{2}}\right)^{2}+q_{3}^{2}}+\sqrt{m^{2}+q_{3}^{2}}\right)^{2}+p_{4}^{2}}, \\
W_{123}^{(\eta)}\left(p_{4}\right) \equiv & \sqrt{\left(\sqrt{\left(2 \sqrt{m^{2}+k_{12}^{2}}\right)^{2}+q_{3}^{2}}+\sqrt{m^{2}+q_{3}^{2}}+w_{123}^{(\eta)}\right)^{2}+p_{4}^{2}} \\
& -\sqrt{\left(\sqrt{\left(2 \sqrt{m^{2}+k_{12}^{2}}\right)^{2}+q_{3}^{2}}+\sqrt{m^{2}+q_{3}^{2}}\right)^{2}+p_{4}^{2}},
\end{aligned}
$$

where $\eta$ can be chosen 1,2 or 3 .

\section{Appendix C}

The boosted potential $V_{12}\left(\vec{k}, \vec{k}^{\prime} ; q\right) \equiv\left\langle\vec{k}\left|\hat{V}_{12}(q)\right| \vec{k}^{\prime}\right\rangle$ of Eq.(18) is a solution of the following quadratic integral equation [7].

$$
\begin{aligned}
V_{12}\left(\vec{k}, \vec{k}^{\prime} ; q\right)= & \frac{1}{\sqrt{4\left(m^{2}+k^{2}\right)+q^{2}}+\sqrt{4\left(m^{2}+k^{\prime 2}\right)+q^{2}}} \\
& \times\left(\left(\sqrt{4\left(m^{2}+k^{2}\right)}+\sqrt{4\left(m^{2}+k^{\prime 2}\right)}\right) v_{12}\left(\vec{k}, \vec{k}^{\prime}\right)\right. \\
& \left.\quad+\int\left(v_{12}\left(\vec{k}, \vec{k}^{\prime \prime}\right) v_{12}\left(\vec{k}^{\prime \prime}, \vec{k}^{\prime}\right)-V_{12}\left(\vec{k}, \vec{k}^{\prime \prime} ; q\right) V_{12}\left(\vec{k}^{\prime \prime}, \vec{k}^{\prime} ; q\right)\right) d^{3} k^{\prime \prime}\right),
\end{aligned}
$$

with

$$
v_{12}\left(\vec{k}, \vec{k}^{\prime}\right) \equiv\left\langle\vec{k}\left|\hat{v}_{12}\right| \vec{k}^{\prime}\right\rangle .
$$

The boosted potential $V_{12}^{[3+1]}\left(\vec{k}, \vec{k}^{\prime} ; q, r\right) \equiv\left\langle\vec{k}\left|\hat{V}_{12}^{[3+1]}(q ; r)\right| \vec{k}^{\prime}\right\rangle$ of Eq. (55) is a solution of the following quadratic integral equation.

$$
\begin{aligned}
& V_{12}^{[3+1]}\left(\vec{k}, \vec{k}^{\prime} ; q, r\right) \\
& =\frac{1}{\sqrt{\left(\sqrt{4\left(m^{2}+k^{2}\right)+q^{2}}+\sqrt{m^{2}+q^{2}}\right)^{2}+r^{2}}+\sqrt{\left(\sqrt{4\left(m^{2}+k^{\prime 2}\right)+q^{2}}+\sqrt{m^{2}+q^{2}}\right)^{2}+r^{2}}} \\
& \times\left(\left(\sqrt{4\left(m^{2}+k^{2}\right)+q^{2}}+\sqrt{4\left(m^{2}+k^{\prime 2}\right)+q^{2}}+2 \sqrt{m^{2}+q^{2}}\right) V_{12}\left(\vec{k}, \vec{k}^{\prime} ; q\right)\right. \\
& \left.\quad+\int\left(V_{12}\left(\vec{k}, \vec{k}^{\prime \prime} ; q\right) V_{12}\left(\vec{k}^{\prime \prime}, \vec{k}^{\prime} ; q\right)-V_{12}^{[3+1]}\left(\vec{k}, \vec{k}^{\prime \prime} ; q, r\right) V_{12}^{[3+1]}\left(\vec{k}^{\prime \prime}, \vec{k}^{\prime} ; q, r\right)\right) d^{3} k^{\prime \prime}\right) .
\end{aligned}
$$

Finally, the boosted 3-body force $W_{123}^{(3)}\left(\vec{k}, \vec{q}, \vec{k}^{\prime}, \vec{q}^{\prime} ; r\right) \equiv\left\langle\vec{k} \vec{q}\left|\hat{W}_{123}^{(3)}(r)\right| \vec{k}^{\prime} \vec{q}^{\prime}\right\rangle$ of Eq.(59) is a solution of the following quadratic integral equation.

$$
=\frac{W_{123}^{(3)}\left(\vec{k}, \vec{q}, \vec{k}^{\prime}, \vec{q}^{\prime} ; r\right)}{\sqrt{\left(\sqrt{4\left(m^{2}+k^{2}\right)+q^{2}}+\sqrt{m^{2}+q^{2}}\right)^{2}+r^{2}}+\sqrt{\left(\sqrt{4\left(m^{2}+k^{\prime 2}\right)+q^{\prime 2}}+\sqrt{m^{2}+q^{\prime 2}}\right)^{2}+r^{2}}}
$$




$$
\begin{aligned}
& \times\left(\left(\sqrt{4\left(m^{2}+k^{2}\right)+q^{2}}+\sqrt{4\left(m^{2}+k^{\prime 2}\right)+q^{\prime 2}}+\sqrt{m^{2}+q^{2}}+\sqrt{m^{2}+q^{\prime 2}}\right) w_{123}^{(3)}\left(\vec{k}, \vec{q}, \vec{k}^{\prime}, \vec{q}^{\prime}\right)\right. \\
& +\iint\left(w_{123}^{(3)}\left(\vec{k}, \vec{q}, \vec{k}^{\prime \prime}, \vec{q}^{\prime \prime}\right) w_{123}^{(3)}\left(\vec{k}^{\prime \prime}, \vec{q}^{\prime \prime}, \vec{k}^{\prime}, \vec{q}^{\prime}\right)-W_{123}^{(3)}\left(\vec{k}, \vec{q}, \vec{k}^{\prime \prime}, \vec{q}^{\prime \prime} ; r\right) W_{123}^{(3)}\left(\vec{k}^{\prime \prime}, \vec{q}^{\prime \prime}, \vec{k}^{\prime}, \vec{q}^{\prime} ; r\right)\right) \\
& \left.\times d^{3} k^{\prime \prime} d^{3} q^{\prime \prime}\right),
\end{aligned}
$$

with

$$
w_{123}^{(3)}\left(\vec{k}, \vec{q}, \vec{k}^{\prime}, \vec{q}^{\prime}\right) \equiv\left\langle\vec{k} \vec{q}\left|\hat{w}_{123}^{(3)}\right| \vec{k}^{\prime} \vec{q}^{\prime}\right\rangle .
$$

These equations from (60) to (63) may be solved by the iteration method [7].

\section{References}

[1] L. D. Faddeev, Scattering theory for a three particle system, Sov. Phys. JETP 12, 1014 (1961).

[2] W. Glöckle, H. Witała, D. Hüber, H. Kamada and J. Golak, The three nucleon continuum: Achievements, challenges and applications, Phys. Rept. 274, 107 (1996), doi:10.1016/0370-1573(95)00085-2.

[3] W. Glöckle, T. S. H. Lee and F. Coester, Relativistic effects in three-body bound states, Phys. Rev. C 33, 709 (1986), doi:10.1103/PhysRevC.33.709.

[4] B. D. Keister and W. N. Polyzou, Relativistic Hamiltonian dynamics in nuclear and particle physics, Adv. Nucl. Phys. 20, 225 (1991).

[5] H. Kamada and W. Glöckle, Momentum transformation connecting a NN potential in the nonrelativistic and the relativistic two nucleon Schrödinger equation, Phys. Rev. Lett. 80, 2547 (1998), doi:10.1103/PhysRevLett.80.2547, arXiv:nucl-th/9903071.

[6] H. Kamada, A practical method for relativistic $3 \mathrm{~N}$-scattering calculations with realistic potentials, Few Body Syst. Suppl. 12, 433 (2000), doi:10.1007/978-3-7091-6287-3_77.

[7] H. Kamada and W. Glöckle, Realistic two-nucleon potentials for the relativistic two-nucleon Schrödinger equation, Phys. Lett. B 655, 119 (2007), doi:10.1016/j.physletb.2007.07.071, arXiv:nucl-th/0703010.

[8] H. Witała, J. Golak, R. Skibiński, W. Glöckle, H. Kamada and W. N. Polyzou, Threenucleon force in relativistic three-nucleon Faddeev calculations, Phys. Rev. C 83, 044001 (2011), doi:10.1103/PhysRevC.83.044001, [Erratum: Phys. Rev. C 88, 069904 (2013), doi:10.1103/PhysRevC.88.069904], arXiv:1101.4053.

[9] W. N. Polyzou, C. Elster, W. Glöckle, J. Golak, Y. Huang, H. Kamada, R. Skibiński and H. Witała, Mini review of Poincaré invariant quantum theory, Few Body Syst. 49, 129 (2011), doi:10.1007/s00601-010-0149-x, arXiv:1008.5215.

[10] H. Kamada, W. N. Polyzou, H. Witała and K. Miyagawa, Lorentz boosted potential for a twobody system with unequal masses, Few Body Syst. 55, 1079 (2014), doi:10.1007/s00601014-0877-4.

[11] B. Bakamjian and L. H. Thomas, Relativistic particle dynamics. II, Phys. Rev. 92, 1300 (1953), doi:10.1103/PhysRev.92.1300. 
[12] A. Arslanaliev, H. Kamada, A. Shebeko, M. Stepanova, H. Witała and S. Yakovlev, Applications of the Kharkov potential in the theory of nuclear forces and nuclear reactions, Prob. Atomic Sci. Technol. 3 (2018).

[13] H. Kamada, W. Glöckle, J. Golak and C. Elster, Lorentz boosted NN potential for few-body systems: Application to the three-nucleon bound state, Mod. Phys. Lett. A 18, 124 (2003), doi:10.1142/S0217732303010089.

[14] H. Kamada, W. Glöckle, H. Witała, J. Golak, R. Skibiński, W. Polyzou and C. Elster, Lorentz boosted nucleon-nucleon T-matrix and the triton binding energy, Mod. Phys. Lett. A 24, 804 (2009), doi:10.1142/S021773230900005X, arXiv:0810.2148.

[15] H. Kamada, O. Shebeko and A. Arslanaliev, Triton binding energy of Kharkov potential, Few Body Syst. 58, 70 (2017), doi:10.1007/s00601-017-1241-2.

[16] K. Sekiguchi et al., Resolving the discrepancy of $135-\mathrm{MeV}$ pd elastic scattering cross sections and relativistic effects, Phys. Rev. Lett. 95, 162301 (2005), doi:10.1103/PhysRevLett.95.162301, arXiv:nucl-ex/0510005.

[17] Y. Maeda et al., Differential cross section and analyzing power measurements for polarized $n(d)$ elastic scattering at 248-MeV, Phys. Rev. C 76, 014004 (2007), doi:10.1103/PhysRevC.76.014004.

[18] H. Witała, J. Golak, R. Skibiński, W. Glöckle, W. N. Polyzou and H. Kamada, Relativity and the low energy nd $A_{y}$ ) puzzle, Phys. Rev. C 77, 034004 (2008), doi:10.1103/PhysRevC.77.034004, arXiv:0801.0367.

[19] O. A. Yakubovsky, On the Integral equations in the theory of $N$ particle scattering, Sov. J. Nucl. Phys. 5, 937 (1967).

[20] R. Fong and J. Sucher, Relativistic particle dynamics and the S matrix, J. Math. Phys. 5, 456 (1964), doi:10.1063/1.1704138.

[21] D. Hüber, H. Kamada, H. Witała and W. Glöckle, How to include a three nucleon force into Faddeev equations for the $3 \mathrm{~N}$ continuum: A New form, Acta Phys. Polon. B 28, 1677 (1997), arXiv:nucl-th/9611007.

[22] H. Kamada, Four-body scattering equations including three-body force in the Faddeev-Yakubovsky theory, Few Body Syst. 60, 33 (2019), doi:10.1007/s00601-019-15014. 